\title{
Avaliação da exposição interna de trabalhadores em serviços de medicina nuclear através da análise de aerossóis contendo ${ }^{131} \mathbf{I}$
}

\author{
Luana Gomes Carneiro ${ }^{a}$, Ana Paula Fonseca de Almeida ${ }^{a}$, \\ Ana Letícia Almeida Dantas ${ }^{\mathrm{a}}$, Eder Augusto Lucena ${ }^{\mathrm{a}}$, Maristela Souza Santos ${ }^{\mathrm{a}}$ e Bernardo \\ Maranhão Dantas ${ }^{a}$ \\ ${ }^{a}$ Instituto de Radioproteção e Dosimetria (IRD-CNEN),Rio de Janeio,Brasil \\ E-mail: carneiro@ird.gov.br
}

\begin{abstract}
RESUMO
Neste estudo foram avaliados os riscos de exposição ocupacional interna associados à incorporação de ${ }^{131}$ I, via inalação, em Serviços de Medicina Nuclear, utilizando técnicas de análise de aerossóis. Os Indivíduos Ocupacionalmente Expostos (IOE) envolvidos na manipulação deste radionuclídeo estão sujeitos à exposição crônica, o que pode levar a um aumento da dose efetiva comprometida. Resultados obtidos em estudos preliminares indicam a ocorrência de incorporação de ${ }^{131}$ I por profissionais envolvidos na manipulação de soluções destinadas a procedimentos de iodoterapia. A avaliação foi realizada no laboratório de radiofarmácia do SMN de um Hospital público localizado na cidade do Rio de Janeiro. Depois de confirmada, através de uma avaliação qualitativa, a presença do radioisótopo, foi determinado um arranjo experimental para a coleta de amostra, tendo sido detectada e quantificada a presença de vapor de ${ }^{131}$ I durante a rotina de trabalho. A concentração média de atividade obtida neste estudo foi de $3 \mathrm{~Bq} / \mathrm{m}^{3}$. Este valor está abaixo Concentração Derivada no $\mathrm{Ar}$ (DAC) de 8,4 x $10^{3} \mathrm{~Bq}$ de ${ }^{131} \mathrm{I} /$ $\mathrm{m}^{3}$ correspondendo a uma dose efetiva comprometida de $1,76 \times 10^{-4} \mathrm{mSv}$. Estes resultados demostram que o ambientes estudados é seguro em termos de exposição interna dos trabalhadores. No entanto, a presença de ${ }^{131}$ I deve ser periodicamente reavaliada, uma vez que este tipo de exposição contribui para o aumento das doses efetivas individuais. Com base nos dados obtidos foram sugeridas melhorias no sistema de exaustão e a utilização de boas práticas de trabalho visando à otimização das exposições.
\end{abstract}

Keywords:

Iodo-131, Aerossol, Medicina Nuclear 


\section{INTRODUCTION}

O Iodo-131 é um radiofármaco de ampla utilização em Medicina Nuclear, onde é utilizado em diversos procedimentos que incluem diagnóstico e tratamento de doenças associadas à tireoide. A manipulação de soluções contendo radioiodo pode resultar em um risco significante de exposição ocupacional devido à possibilidade de incorporação via inalação ou ingestão. [1;2;3]

Diversos estudos sobre a exposição ocupacional em medicina nuclear tem sido publicados onde o aumento na utilização de radioisótopos e disponibilidade de metodologias apropriadas para avaliação da dose individual tem sido amplamente discutidas. Um estudo preliminar incluindo avaliação in vivo e in vitro, realizado por Lucena et al., em 2007 [4], indicou a ocorrência de incorporação por trabalhadores envolvidos na manipulação de soluções contendo radioiodo, utilizadas na iodoterapia.[5;6;7;8;9;10;11;12]

A Agência Internacional de Energia Atômica (AIEA) em seu Guia de Segurança N ${ }^{\circ}$ RS-G-1.2 recomenda que a monitoração ocupacional seja implementada sempre que houver risco de dose efetiva anual decorrente de incorporações de radionuclídeos superar o valor de $1 \mathrm{mSv}$. A inalação está entre as principais rotas de entrada de radionuclídeos no corpo humano e a principal rota de exposição ocupacional a ${ }^{131} \mathrm{I}$, neste caso a avaliação do ambiente através da análise de aerossóis é recomendado por este documento. [1].

O iodo é um elemento químico volátil à temperatura ambiente, sendo liberado na forma de vapor, que passa livremente através dos poros de filtros comuns, impossibilitando que estes sejam utilizados para em sua retenção. Neste caso, diversos materiais adsorventes são utilizados para a retenção do elemento.

Diversas técnicas para a investigação da presença de ${ }^{131}$ I têm sido estudadas e testadas por diversos grupos de pesquisa. O carvão ativo é um dos substratos mais utilizados para adsorver o iodo. Esta adsorção é favorecida pela natureza intrínseca do substrato, sua alta porosidade e a grande área superficial, decorrentes do processo de preparo do carvão.

Em 2010 Ramos et al. testaram a eficiência do carvão ativo para a adsorção do $\mathrm{I}_{2}$, uma das forma mais comuns de iodo volátil encontrado nos Serviços de Medicina Nuclear (SMNs), constatando uma eficiência de $100 \%$ de retenção desta forma química. Segundo Sales (1981), [13] o uso do carvão como meio filtrante deve ser controlado devido ao seu baixo ponto de ignição, ou seja, quanto menor a quantidade de carvão, maior a segurança [14].

A avaliação do ar no ambiente de trabalho pode fornecer importantes informações para o controle da exposição de trabalhadores envolvidos na manipulação de ${ }^{131} \mathrm{I}$, tornando possível estabelecer soluções para a melhoria das condições de trabalho. 
O objetivo principal deste trabalho foi o desenvolvimento de uma metodologia para a realização de medidas com filtros contendo carvão ativado, preparados para avaliar a presença de ${ }^{131}$ I no ar em Serviços de Medicina Nuclear da cidade do Rio de Janeiro, e, consequentemente, a possibilidade de incorporação deste elemento por trabalhadores envolvidos em sua manipulação.

\section{MATERIAIS E MÉTODOS}

O estudo para o desenvolvimento da metodologia proposta foi realizado no Serviço de Medicina Nuclear de um Hospital Público localizado na cidade do Rio de Janeiro, que aceitou voluntariamente participar da pesquisa.

Neste SMN o ${ }^{131}$ I é manipulado na forma de capsulas e soluções líquidas. A manipulação de cápsulas, a princípio, representa um baixo risco de exposição interna aos trabalhadores envolvidos, portanto, o presente estudo ficou restrito ao laboratório de radiofarmacia onde são fracionadas as doses de ${ }^{131} \mathrm{I}$ a serem administradas nos pacientes.

Neste local realizou-se, em um período pré-determinado, amostragens de ar para a avaliação da presença de ${ }^{131}$ I no ambiente durante a rotina de trabalho e, uma vez identificada a presença deste radioisótopo, foi otimizado o arranjo experimental de coleta de amostra de aerossóis nos laboratórios de radiofarmacia.

Foram realizados quatro ensaios e foram obtidos 16 filtros que foram analisados por espectrometria gama com detector semicondutor HPGe disponível no Laboratório de Bioanálise in vitro da Divisão de Dosimetria (DiDos) do Instituto de Radioproteção e Dosimetria (IRD).

\subsection{PREPARO DOS FILTROS E ARRANJO EXPERIMENTAL}

O arranjo experimental proposto neste trabalho foi desenvolvido de forma a causar o mínimo possível de incômodo na rotina de trabalho, considerando as dimensões, geralmente pequenas, das salas de manipulação de radiofármacos e o nível de concentração e cuidados que este tipo de trabalho exige.

A avaliação da presença de vapor de ${ }^{131}$ I foi realizada utilizando amostradores portáteis SKC do tipo "Samplers Airchek" modelo 224-44XR e holders de 37mm de diâmetro com filtros contendo carvão ativado preparados no Laboratório de Caracterização de Aerossóis (LCA) da Divisão de Dosimetria do Instituto de Radioproteção e Dosimetria.

Os filtros, denominados "sanduiche de carvão ativo" foram confeccionados utilizando os seguintes materiais: filtros de fibra de vidro de $37 \mathrm{~mm}$ de diâmetro, carvão ativo com 
granulometria < que $100 \mu \mathrm{m}$ e cola branca. Para cada filtro foram pesados 0,15 mg de carvão sobre um filtro de fibra de vidro, e fechado em uma espécie de sanduiche com o uso de cola branca aplicada na borda do filtro superior. Após a secagem, os sanduiches já prontos são colocados em um holder do mesmo tipo utilizado em amostradores portáteis e este sistema é acoplado a uma bomba de sucção de baixa vazão. Na fig. 1 é apresentado o "Samplers Airchek" modelo 224-44XR e o holder.

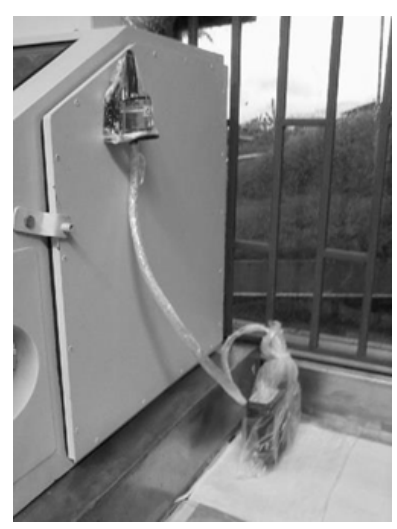

Figura 1: Amostrador SKC “Samplers Airchek" modelo 224-44XR e holder.

O arranjo experimental utilizado neste estudo consiste no posicionamento de quatro amostradores, sendo dois do lado direito e dois do lado esquerdo da capela onde ocorre a manipulação da solução de ${ }^{131} \mathrm{I}$. Em cada lado os amostradores foram posicionados imediatamente ao lado da entrada da capela e à um metro de distância da capela, ambos posicionados à um metro e meio de altura em relação ao chão da sala. Este arranjo experimental pode ser visto nas figuras 2 e 3 .

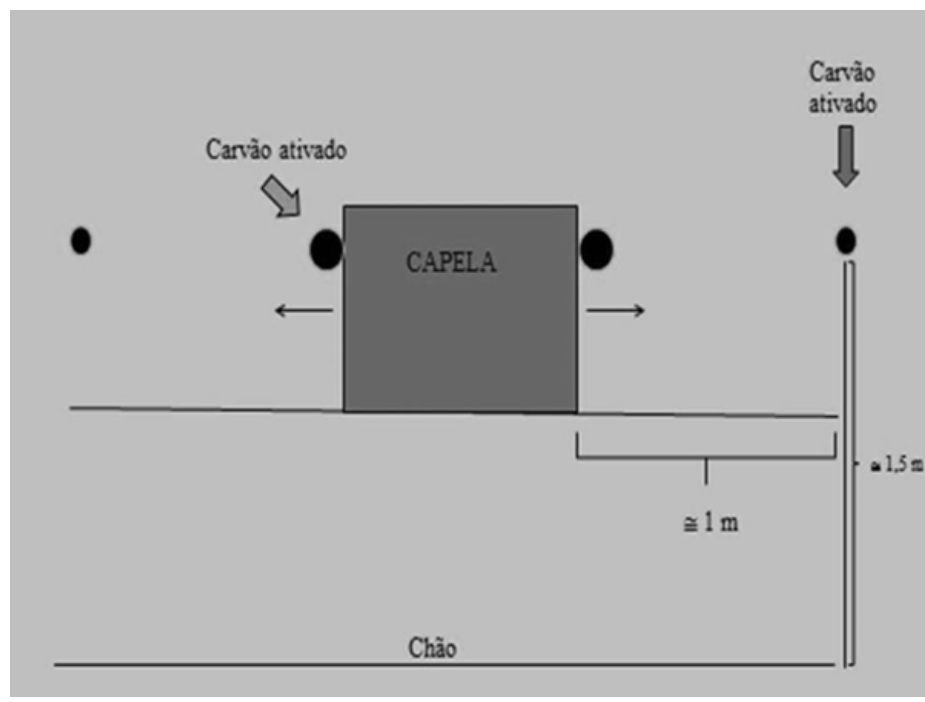


Figura 2: Desenho esquemático do arranjo experimental
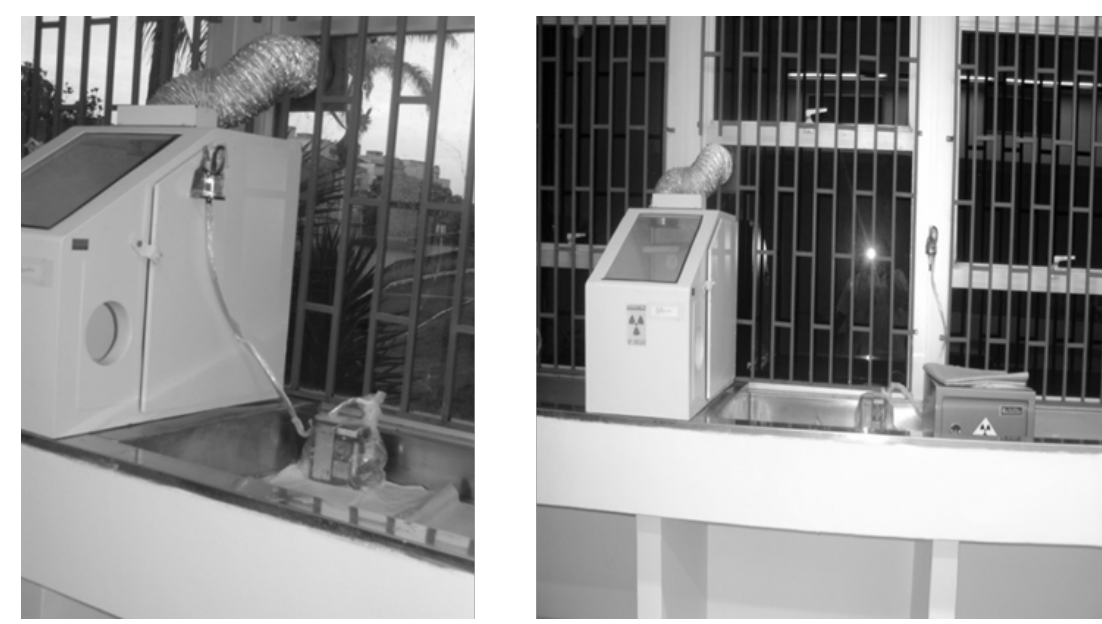

Figura 3: Posicionamento do sistema de amostragem do arranjo experimental

\subsection{CALIBRAÇÃO DOS SISTEMAS DE DETECÇÃO PARA DETERMINAÇÃO DE ${ }^{131}$ I NAS AMOSTRAS DE AR}

As amostras foram analisadas utilizando o sistema de detecção de Germânio Hiperpuro (HPGe) coaxial da marca Camberra ${ }^{\circledR}$ disponível no Laboratório de Bioanálises In Vitro da Divisão de Dosimetria do IRD. O programa utilizado na análise dos espectros foi o Genieß-2000, também fornecido pela empresa Canberra ${ }^{\circledR}$.

Para a calibração do sistema de detecção foi utilizada uma solução-padrão líquida de ${ }^{133}$ Ba com atividade certificada pelo Laboratório Nacional de Metrologia das Radiações Ionizantes/LNMRI/IRD. Foi padronizada a geometria de contagem para o sanduíche de carvão, a fonte para calibração foi preparada utilizando o método de deposição gravimétrica com picnômetro. A fonte foi preparada usando um sanduíche contendo 0,15 g de carvão entre dois filtros de fibra de vidro. A solução foi gotejada seguindo um modelo geométrico baseado no esquema geométrico hexagonal desenvolvido por Ceccatelli et al (2010),[15] de forma a encontrar uma distribuição mais homogênea possível. [16] 
Após o preparo, as fontes passaram pela secagem em lâmpada infravermelho, foram seladas com lâmina adesiva de polietileno (tipo contact) e acopladas a um holder de $37 \mathrm{~mm}$ idêntico ao utilizado durante as amostragens. Cada fonte foi submetida a 10 medições com tempo fixo. Para realização dos cálculos foi utilizada a média das contagens.

Para correção da atividade presente depositada no filtro foi utilizada a equação 1:

$$
A=A_{0} x e^{-\lambda t}
$$

A atividade equivalente de ${ }^{131}$ I foi calculada segundo a equação 2:

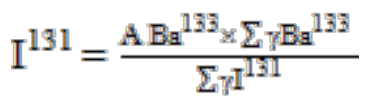

O fator de calibração foi obtido segundo a equação 3:

$$
\mathrm{F}_{\mathrm{c}}=\frac{\mathrm{cpm}(\text { filtro) }}{\mathrm{A}_{\mathrm{ralib}}}
$$

Onde,

cpm - Taxa de contagem líquida do filtro (total de contagens na região de interesse subtraída da radiação de fundo)

A - atividade equivalente de ${ }^{131}$ I presente no filtro no momento da medição

A partir dos dados obtidos no processo de calibração é possível calcular as atividades e as concentrações de ${ }^{131}$ I no ar no ambiente avaliado. 


\section{RESULTADOS E DISCUSSÕES}

A concentração de radionuclídeos no ar é avaliada em termos da Concentração Derivada no Ar (Derived Air Concentration - DAC) que é definida como a concentração em atividade no ar que resultará em uma incorporação de $\mathrm{I}_{\text {inhl,L }}$ por um trabalhador continuamente exposto por um ano. Considerando que a taxa de respiração do Homem Referência da ICRP é de $1.2 \mathrm{~m} 3 / \mathrm{h}$ para $2000 \mathrm{~h}$ de trabalho por ano, a DAC para 131I na forma de vapor é $416.67 \mathrm{~Bq} / \mathrm{m}^{3}$.

As medidas foram realizadas utilizando o filtro contendo carvão ativado (sanduíche) preparado no Laboratório de Caracterização de Aerossol do IRD. As amostragens foram realizadas na vazão de operação de $1 \mathrm{~L} / \mathrm{min}$.

Os resultados obtidos estão apresentados na tabela 1. 
Tabela 1: Resultados das amostragens

\begin{tabular}{|c|c|c|c|}
\hline Amostra & Ponto de coleta & $\begin{array}{c}\text { Atividade } \\
\text { no filtro } \\
\text { (Bq) }\end{array}$ & $\begin{array}{c}\text { Concentração } \\
\text { no filtro } \\
\left(\mathbf{B q} / \mathbf{m}^{3}\right)\end{array}$ \\
\hline 1 & Direita (face externa capela) & $7,5 \mathrm{E}+00$ & $1,9 \mathrm{E}+01$ \\
\hline 2 & Esquerda(face externa capela) & $1,7 \mathrm{E}+00$ & $4,4 \mathrm{E}+00$ \\
\hline 3 & Direita (1m da capela) & $2,3 \mathrm{E}+00$ & $5,8 \mathrm{E}+00$ \\
\hline 4 & Esquerda (1m da capela) & $2,3 \mathrm{E}+00$ & $5,9 \mathrm{E}+00$ \\
\hline 5 & Direita (1m da capela) & 4,5E-03 & 3,0E-02 \\
\hline 6 & Direita(face externa capela) & 2,5E-02 & $1,4 \mathrm{E}-01$ \\
\hline 7 & Esquerda (face externa capela) & 7,5E-03 & 4,5E-02 \\
\hline 8 & Esquerda (1m da capela) & 7,4E-03 & 5,3E-02 \\
\hline 9 & Direita (1m da capela) & 1,33E-02 & $3,4 \mathrm{E}-02$ \\
\hline 10 & Direita(face externa capela) & 3,22E-03 & 8,2E-03 \\
\hline 11 & Esquerda (face externa capela) & 1,20E-02 & 2,6E-02 \\
\hline 12 & Esquerda (1m da capela) & 3,91E-03 & 7,7E-03 \\
\hline 13 & Direita (1m da capela) & $<\mathrm{AMD}^{*}$ & $<\mathrm{AMD}^{*}$ \\
\hline 14 & Direita(face externa capela) & $<\mathrm{AMD}$ & $<\mathrm{AMD}$ \\
\hline 15 & Esquerda (face externa capela) & $<\mathrm{AMD}$ & $<\mathrm{AMD}$ \\
\hline 16 & Esquerda (1m da capela) & $<\mathrm{AMD}$ & $<\mathrm{AMD}$ \\
\hline Média & & $3 \mathrm{~Bq} / \mathrm{m}^{3}$ & \\
\hline
\end{tabular}

*AMD = 1,60E-02 Bq

O valor de DAC de $416.67 \mathrm{~Bq} / \mathrm{m}^{3}$ para ${ }^{131} \mathrm{I}$ na forma de vapor considera uma rotina de trabalho de 8 horas por dia, 5 dias por semana, ou 2000 horas por ano, sendo os trabalhadores 
constantemente expostos durante este período. Contudo, os trabalhadores envolvidos na manipulação de radiofármacos não cumprem esta rotina de trabalho, havendo um rodízio e um tempo mínimo de manipulação para reduzir ao máximo a exposição.

Com base nesta rotina diferenciada de trabalho foram calculados valores de DAC para diferentes tempos de exposição, considerando a manipulação ocorrendo duas vezes na semana, o tempo observado no SMN estudado e o relato dos trabalhadores. Na tabela 2 são apresentados os dados utilizados e as concentrações estimadas para diferentes condições de trabalho considerando as médias obtidas em cada dia de amostragem.

Tabela 2: Estimativa da DAC para diferentes tempos de exposição

\begin{tabular}{c|ccccccc}
\hline $\begin{array}{c}\text { Tempo de } \\
\text { exposição } \\
\text { (min) }\end{array}$ & $\begin{array}{c}\text { Tempo de } \\
\text { exposição } \\
\text { (h) }\end{array}$ & $\begin{array}{c}\text { Tempo } \\
\text { por } \\
\text { semana }\end{array}$ & $\begin{array}{c}\text { Exposição } \\
\text { em um } \\
\text { ano }\end{array}$ & $\begin{array}{c}\text { DAC } \\
\left.\mathbf{( B q} / \mathbf{m}^{3}\right)\end{array}$ & $\mathbf{2 1 / 1 0 / 2 0 1 3}$ & $\mathbf{2 8 / 1 0 / 2 0 1 3}$ & $\mathbf{1 1 / 1 1 / 2 0 1 3}$ \\
\hline 10 & 0,17 & 0,33 & 17,33 & $4,86 \mathrm{E}+04$ & $2,57 \mathrm{E}+01$ & $1,75 \mathrm{E}-01$ & $5,49 \mathrm{E}-02$ \\
20 & 0,33 & 0,67 & 34,67 & $2,43 \mathrm{E}+04$ & $5,14 \mathrm{E}+01$ & $3,51 \mathrm{E}-01$ & $1,10 \mathrm{E}-01$ \\
30 & 0,50 & 1,00 & 52,00 & $1,62 \mathrm{E}+04$ & $7,71 \mathrm{E}+01$ & $5,26 \mathrm{E}-01$ & $1,65 \mathrm{E}-01$ \\
40 & 0,67 & 1,33 & 69,33 & $1,21 \mathrm{E}+04$ & $1,03 \mathrm{E}+02$ & $7,01 \mathrm{E}-01$ & $2,20 \mathrm{E}-01$ \\
50 & 0,83 & 1,67 & 86,67 & $9,71 \mathrm{E}+03$ & $1,29 \mathrm{E}+02$ & $8,77 \mathrm{E}-01$ & $2,74 \mathrm{E}-01$ \\
60 & 1,00 & 2,00 & 104,00 & $8,09 \mathrm{E}+03$ & $1,54 \mathrm{E}+02$ & $1,05 \mathrm{E}+00$ & $3,29 \mathrm{E}-01$ \\
70 & 1,17 & 2,33 & 121,33 & $6,94 \mathrm{E}+03$ & $1,80 \mathrm{E}+02$ & $1,23 \mathrm{E}+00$ & $3,84 \mathrm{E}-01$ \\
80 & 1,33 & 2,67 & 138,67 & $6,07 \mathrm{E}+03$ & $2,06 \mathrm{E}+02$ & $1,40 \mathrm{E}+00$ & $4,39 \mathrm{E}-01$ \\
90 & 1,50 & 3,00 & 156,00 & $5,40 \mathrm{E}+03$ & $2,31 \mathrm{E}+02$ & $1,58 \mathrm{E}+00$ & $4,94 \mathrm{E}-01$ \\
100 & 1,67 & 3,33 & 173,33 & $4,86 \mathrm{E}+03$ & $2,57 \mathrm{E}+02$ & $1,75 \mathrm{E}+00$ & $5,49 \mathrm{E}-01$ \\
110 & 1,83 & 3,67 & 190,67 & $4,41 \mathrm{E}+03$ & $2,83 \mathrm{E}+02$ & $1,93 \mathrm{E}+00$ & $6,04 \mathrm{E}-01$ \\
120 & 2,00 & 4,00 & 208,00 & $4,05 \mathrm{E}+03$ & $3,09 \mathrm{E}+02$ & $2,10 \mathrm{E}+00$ & $6,59 \mathrm{E}-01$ \\
\hline
\end{tabular}




\section{CONCLUSÕES}

De acordo com os dados é possível observar que as concentrações estimadas para um ano de trabalho baseadas nas concentrações medidas neste estudo considerando diferentes tempos de exposição estão todas abaixo dos valores de DAC. Entretanto, é importante levar em consideração que estes trabalhadores estão sujeitos de forma crônica tanto à exposição interna quanto à externa e também a outros radiofármacos, sendo recomendável, se possível e economicamente viável, reduzir todos os tipos de exposição a valores tão baixos quanto possível.

Os procedimentos para coleta e analises radiométricas desenvolvidos neste estudo mostraram-se adequados para o objetivo proposto, tanto do ponto de vista da praticidade na implementação quanto na sensibilidade necessária para uma avaliação da exposição ocupacional.

Com os resultados obtidos é possível concluir que, no ambiente estudado, ocorre volatilização do

${ }^{131}$ I durante a manipulação. A exposição foi confirmada através da medida de tireoide e urina dos envolvidos na manipulação, reforçando a sensibilidade do método a este nível de exposição.

Os resultados da estimativa do DAC para diferentes condições de trabalho sugerem a possibilidade de otimização da exposição. Assim, recomenda-se, na medida do possível, a adoção de rodízio de funcionários na rotina de manipulação do elemento.

Estudos complementares são necessários para avaliar se a presença do ${ }^{131} \mathrm{I}$ no ar e sua incorporação estão associadas a boas práticas, estrutura e projeto da sala de manipulação ou somente às características do radionuclídeo.

\section{AGRADECIMENTO}

Os autores agradecem ao CNPq pelo suporte financeiro para disponibilizado, Agradecemos especialmente ao Diretor do Hospital, à Chefia do Serviço de Medicina Nuclear e aos profissionais que participaram voluntariamente e gentilmente desta pesquisa. 


\section{REFERÊNCIAS}

1. International Atomic Energy Agency (IAEA). Assessment of Occupational Exposure Due to Intakes of Radionuclides. Safety Standards Series, $n^{0}$. RS-G-1.2, IAEA (1999).

2. I.G. Schwartz, W.T. Phillips, C.A. Shriver. "The risk of radiation exposure to laboratory personnel”. Lab med. Vol.22, n 2, pp.114-119 (1991).

3. C.C. Albino, M.H. Takhashi, S.S. Junior, H.Graf. "Inquérito sobre o uso do Iodo-131 no Brasil”. Arq Bras. Endocrinol. Metab., Vol. 45, n 6, pp 558-562 (2001)

4. E. A. Lucena, A. M. O. Rebelo, F. Araujo, W. O. Sousa, A. L. A. Dantas, B. M. Dantas, R. Corbo. "Evaluation of internal exposure of nuclear medicine staff through in vivo and in vitro bioassay techniques”. Radiat. Prot. Dosimetry. Vol. 127, pp. 465-468, (2007).

5. S. Baechler, N. Stritt e , F. O. Bochud.” Individual monitoring of internal exposure for nuclear medicine workers in Switzerland”. Radiation Protection Dosimetry, 144(1-4): 464467 (2011).

6. J. Bento, P. Teles, M. Neves, A. I. Santos, G. Cardoso, A. Barreto, F. Alves, C. Guerreiro, A. Rodrigues, J. A. M. Santos, C. Capelo, R. Parafita, B. Martins. "Study of nuclear medicine practices in Portugal from an internal dosimetry perspective”. Radiation Protection Dosimetry. Vol. 149, pp 438-443 (2012).

7. B. M. Dantas, E. A. Lucena, A. L. A. Dantas, F. Araujo, A. M. O. Rebelo, M. Teran, A. Paolino, J.C. Hermida, A. M. Rojo, J. A. Puerta, J. Morales, G.M.L. Bejerano, M. Alfaro, M. A. Ruiz,R. Videla, O. Pinones, S. Gonzalez, T. Navarro, D. Melo, R. Cruz-Suarez. “A protocol for the calibration of gamma cameras to estimate internal contamination in emergency situations”. Radiat Prot Dosimetry. Vol. 127, pp 253-257 (2007).

8. J. Dumeau, J-L. Rehel, B. Aubert. "Evaluation de la contamination atmospherique dans les services de medecine nucleaire.” IRSN. (2011).

9. S.L.Heller."Radiation safety in the central radiopharmacy”. Seminars In Nuclear Medicine. Vol. 26, n 2, pp 107-118. (1996).

10. S.E. Jansen, A. Vanaswegen, M.G. Lotter et al. "Staff radiation-doses during 8 years in a nuclear-medicine radiopharmacy.” Nucl Med Commun. Vol. 15, n 2, pp 114-118. (1994).

11. K. Kletter, A. Becherer, M. Diemling et al. "Radiation burden for the staff from pet and conventional nuclear medicine”. Eur J Nucl Med. Vol.26, n 9, OS358 (1999). 
12. M.S. Vidal, B.M. Dantas, A.L.A. Dantas. "A methodology for auto-monitoring of internal contamination by I-131 in nuclear medicine workers”. Radiation Protection Dosimetry. Vol. 125, n 1-4, pp. 483-487 (2007).

13. F. Gazelli Sales. "Investigações sobre a remoção de compostos de iodo penetrantes na forma gasosa em materiais absorvedores”. Tese de Mestrado, Departamento de Energia Nuclear UFPE (1981).

14. V.S. Ramos, V.R. Crispim, L.E.B. Brandão. "New filter for iodine applied in nuclear medicine services”. Applied Radiation and Isotopes. Vol. 82, pp. 111-118. (2013).

15. A. Ceccatelli, P. De Felice, A. Fazio. "Development of simulated air filters for gamma-ray spectrometry proficiency testing”. Applied Radiation and Isotopes. Vol. 68, pp.1240-1246. (2010).

16. R. C. Mcfarland. "Geometric consideration in the calibration of germanium detectors for filter-paper counting”. Radioact. Radiochem.Vol. 2, n 1. (1991). 